\title{
Peroxisome proliferator-activated receptor gamma modulation and lipogenic response in adipocytes of small-for-gestational age offspring
}

Jennifer K Yee ${ }^{*}$, Wai-Nang Paul Lee ${ }^{1}$, Michael G Ross², Robert H Lane ${ }^{3}$, Guang Han², Juan Vega ${ }^{1}$ and Mina Desai

\begin{abstract}
Background: Small-for-gestational age (SGA) at birth increases risk of development of adult obesity and insulin resistance. A model of SGA rat offspring has been shown to exhibit increased adipose tissue expression of a key adipogenic transcription factor, peroxisome proliferator-activated receptor gamma (PPARY), and increased fatty acid de novo synthesis during the nursing period, prior to onset of obesity. PPARy agonists have been studied for potential use in the prevention of insulin resistance. Moreover, SGA adipocytes exhibit age-dependent differences in lipogenesis as mediated by PPARY. The effects of PPARY modulators on lipogenic gene expression and de novo lipogenesis on the age-dependent changes in SGA adipocytes are not known. The objectives of this study were: 1) to determine the adipogenic and lipogenic potential in SGA adipocytes at postnatal day 1 (p1) and day 21 (p21), 2) to determine how the PPARY activator- and repressor-ligands affect the lipogenic potential, and 3) to determine the fatty acid metabolic response to PPARY activator-ligand treatment.

Methods: Primary adipocyte cultures from p1 and p21 SGA and Control male offspring were established from a known maternal food-restriction model of SGA. Cell proliferation and Oil Red O (ORO) staining were quantified. Adipocytes were treated with increasing doses of rosiglitazone or bisphenol-A diglycidyl ether (BADGE). PPARY and SREBP1 protein expression were determined. De novo lipogenesis with rosiglitazone treatment at p21 was studied using $50 \% \mathrm{U}^{13} \mathrm{C}$-glucose and gas chromatography/mass spectrometry.

Results: At p1 and p21, SGA demonstrated increased cell proliferation and increased ORO staining. At p21, SGA demonstrated increased lipogenic gene expression and increased glucose-mediated fatty acid de novo synthesis compared with Controls. In response to rosiglitazone, SGA adipocytes further increased glucose utilization for fatty acid synthesis. SGA lipogenic gene expression demonstrated resistance to BADGE treatment.

Conclusions: SGA adipocytes exhibit an enhanced adipogenic and lipogenic potential in early postnatal life. By p21, SGA demonstrated resistance to PPARy repressor-ligand treatment, and selective response to high dose PPARY activator-ligand treatment in adipogenic and lipogenic gene expression. p21 SGA adipocytes revealed increased fatty acid de novo synthesis through a complex relationship with glucose metabolism.
\end{abstract}

Keywords: Adipocyte, Small-for-gestational age, Rosiglitazone, PPARY, Stable isotope, Fatty acid, Adipogenesis

\footnotetext{
* Correspondence: jyee@labiomed.org

'Department of Pediatrics, Division of Endocrinology, Los Angeles Biomedical

Research Institute at Harbor-UCLA Medical Center, David Geffen School of

Medicine at UCLA, 1000 West Carson Street, Harbor Box 446, Torrance, CA

90509, USA

Full list of author information is available at the end of the article
} 


\section{Introduction}

Human epidemiological studies have shown that smallfor-gestational age (SGA) newborns demonstrate increased risk for adult obesity and metabolic syndrome $[1,2]$. Animal studies have effectively replicated this association [3-5] in that maternal undernutrition during rat pregnancy results in SGA. When these SGA pups are nursed normally, they exhibit hyperphagia [6], catch-up growth, and develop adult obesity and insulin resistance $[4,5]$. The increased adiposity has been attributed to "programmed" upregulation of the adipogenic transcription factor peroxisome proliferator-activated receptor $\gamma$ (PPAR $\gamma$ ). SGA newborns demonstrate increased PPAR $\gamma$ expression prior to the onset of obesity [7]. The downstream lipid target of PPARY is a lipogenic transcription factor, SREBP1c (sterol regulatory element binding protein 1), which in turn induces expression of lipogenic enzyme fatty acid synthase (FASN) [8]. Both SREBP1c and FASN are upregulated in SGA adipose tissue at the end of the nursing period (p21) [7]. Hence, modulation of PPARY may provide a therapeutic strategy in preventing adiposity in SGA offspring.

As an enhancer of adipocyte differentiation and lipid accumulation, PPAR $\gamma$ has been studied as a target for pharmacological therapy. Rosiglitazone, which is a member of the thiazolidinedione (TZD) class of drugs, is a selective ligand of PPAR $\gamma$. It has the ability to activate PPAR $\gamma$, and hence induce adipocyte differentiation in cell culture models and promote weight gain in rodents and humans $[9,10]$. Recent studies have further shown that rosiglitazone may directly induce expression of PPARy $[11,12]$. Although rosiglitazone causes weight gain, longterm treatment leads to a smaller adipocyte phenotype, suggesting changes in the metabolic pathways leading to fat accumulation [13]. While studies have demonstrated that TZDs increase free fatty acid uptake in adipocytes [14,15], the effects of TZDs on endogenous fatty acid synthesis have not been well-studied. Conversely, bisphenol-A diglycidyl ether (BADGE), a selective PPARY antagonist, has shown the ability to prevent adipocyte differentiation in vitro [16]. BADGE also has the ability to interfere with rosiglitazone-mediated effects on PPAR $\gamma$ since both PPARY modulators (rosiglitazone and BADGE) bind at the ligand-binding domain $[16,17]$.

Consistent with upregulated PPAR $\gamma$, SREBP1c, and lipogenic target genes, our rat model of SGA offspring exhibits increased adipose tissue fatty acid de novo synthesis as early as 3 weeks of age [3]. Thus, SGA offspring demonstrate upregulation of the adipogenesis signaling cascade and enhanced adipose tissue lipogenesis prior to development of an obese phenotype. We hypothesized that the increased adipogenic potential of SGA offspring is an intrinsic cellular response, which contributes to enhanced lipogenesis. Furthermore, we hypothesized that the enhanced lipogenesis is facilitated by increased glucose-mediated fatty acid synthesis. We addressed our hypotheses by utilizing primary adipocyte cultures from 1 day old (p1) and 3 week old (p21) SGA offspring. We further examined the effect of PPAR $\gamma$ modulators, rosiglitazone (activator-ligand) and BADGE (repressor-ligand) on the lipogenic targets downstream of PPAR $\gamma$. To delineate the specific effects of rosiglitazone treatment in the fatty acid synthesis pathways, we studied rosiglitazone-stimulated glucose utilization toward de novo fatty acid synthesis using $\mathrm{U}^{13} \mathrm{C}$-glucose as a stable isotope tracer.

\section{Methods}

\section{Animal studies}

Studies received approval from the Animal Care Committee at the Los Angeles Biomedical Research Institute at Harbor-UCLA and were in accordance with the American Accreditation Association of Laboratory Care.

A description of the maternal food-restriction rat model has been previously published [4]. In brief, firsttime pregnant Sprague-Dawley rats (Charles River Laboratories, Hollister, CA) were housed in an animal facility with controlled 12/12 hour light/dark cycles, and constant temperature and humidity conditions. On day 10 of gestation (e10), dams were either given a standard laboratory ad libitum chow diet (Lab Diet 5001, Brentwood, Missouri) to yield normal size pups, or were $50 \%$ food-restricted to produce SGA pups. Dams continued the assigned diets during pregnancy and lactation. After birth, on day 1 (p1), individual pups were weighed. To standardize nursing, litters were culled to 8 pups per dam (4 males and 4 females) to include the offspring of median weights. SGA offspring were cross-fostered to ad libitum-fed control dams. To control for crossfostering technique, the control pups were also crossfostered to control dams.

Male offspring ( $\mathrm{n}=6$ at each age per group) were decapitated at postnatal day $1(\mathrm{p} 1)$, or given an overdose of pentobarbital $(200 \mathrm{mg} / \mathrm{kg}$ intraperitoneally) at day 21 (p21), then the adipose tissue was collected. Subcutaneous adipose tissue was collected at p1 due to minimal retroperitoneal adipose tissue availability at this age. Retroperitoneal adipose tissue was collected at p21 because previous data on this model already demonstrated abnormalities in this tissue [3].

\section{Primary adipocyte cell cultures}

Pooled adipose tissue from p1 or p21 animals was minced, and digested with collagenase type II $(5000 \mathrm{U} / \mathrm{g})$ in Krebs-Ringer solution for $30 \mathrm{~min}$ at $37{ }^{\circ} \mathrm{C}$, filtered through $200 \mu \mathrm{m}$ mesh nylon filter, then centrifuged at $500 \mathrm{x} \mathrm{g}$ for 5 minutes. The cells were resuspended in 
high glucose $(450 \mathrm{mg} / \mathrm{dl})$ Dulbecco's modified Eagle's medium (DMEM) (Invitrogen) with 10\% fetal bovine serum (FBS) and 1\% Antibiotic-Antimycotics (Invitrogen), seeded into flasks and incubated at $37{ }^{\circ} \mathrm{C}$ with $5 \%$ $\mathrm{CO} 2$ until confluent. For all experiments except for the cell proliferation assay, adipocyte differentiation was induced with dexamethasone $(1 \mu \mathrm{M})$, methylisobutylxanthine $(0.1 \mathrm{mM})$, and insulin $(10 \mu \mathrm{g} / \mathrm{ml})$ for 10 days.

\section{Cell proliferation assay}

Preadipocytes from p1 and p21 Control and SGA male offspring were isolated as above, and seeded $\left(1 \times 10^{4}\right.$ cells $)$ in DMEM supplemented with 10\% FBS and 1\% AntibioticAntimycotics. After 24 hours, 3-(4,5-Dimethylthiazol-2-yl)2,5-diphenyltetrazolium bromide (MTT, from Sigma) was added to each well, then cells were incubated at $37^{\circ} \mathrm{C}$. The remaining MTT was removed, the dye crystals were solubilized in isopropanol, and the absorbance was read by spectrophotometry at $570 \mathrm{~nm}$ wavelength (Perkin Elmer 1420 Mulitlabel counter VICTOR ${ }^{3}$ spectrophotometer).

\section{Oil red $\mathrm{O}(\mathrm{ORO})$ staining and assay}

Preadipocytes were seeded $\left(1 \times 10^{4}\right.$ cells $)$ and cells were allowed to differentiate into mature adipocytes after induction as above. Cells were subsequently fixed with $4 \%$ paraformaldehyde, stained with $0.5 \%$ ORO, mounted onto slides with Vectashield mounting medium with 4',6-diamidino-2-phenylindole (DAPI; Vector) and images (40X magnification) captured (Zeiss Axioskop 40 microscope with Axiocam HRc camera).

For quantification, adipocytes stained with ORO were dried for 1 hour at $37{ }^{\circ} \mathrm{C}$, incubated with a fixed volume of isopropanol for 20 minutes to elute the ORO, and absorbance measured at $570 \mathrm{~nm}$.

\section{PPAR $\gamma$ modulation studies}

After differentiation, adipocytes at p1 and p21 were incubated in high glucose DMEM with rosiglitazone (Sigma) at 0,1 or $10 \mu \mathrm{M}$ concentration, or with BADGE (Alexis Biochemicals) at 0,1 or $10 \mu \mathrm{M}$ concentration. Untreated cells received DMSO for 24 hours. Protein was extracted by sonication in RIPA buffer with phosphodiesterase inhibitor and EDTA, then the concentration was measured using the BCA Protein Assay (Pierce). $20 \mu \mathrm{g}$ of protein was loaded on a precast BioRad BisTris gel, then transferred to nitrocellulose membranes. Non-specific binding was blocked by nonfat milk. Primary antibodies were applied overnight, membranes washed, and species-appropriate secondary antibodies applied. Autoradiography of membranes with chemiluminescence (Pierce Super Signal West Pico Chemiluminescence kit) was carried out in a darkroom. After stripping and washing of the original membrane, a primary antibody suitable for use as an internal control was applied, followed by a secondary antibody and chemiluminescence. Densitometry was performed on the protein of interest and band intensities were normalized to those of the corresponding control. The following are the antibodies used and their respective sources: PPAR $\gamma$ (Affinity Bio Reagents, AB Cam), SREBP1 (Santa Cruz), FASN (Santa Cruz).

\section{Stable isotope fatty acid metabolism studies}

Primary adipocytes from p21 male RP fat were isolated and differentiated as above. For 24 hours following differentiation, Control and SGA adipocytes received DMSO or rosiglitazone $10 \mu \mathrm{M}(\mathrm{n}=3$ each group). $50 \%$ of the glucose in the high glucose DMEM was comprised of $\mathrm{U}^{13} \mathrm{C}$-glucose as a tracer. After this experimental period, cells from each flask were collected as pellets for fatty acid analysis and Western blotting.

\section{Fatty acid extractions}

Total fatty acids (from phospholipids, triglycerides, cholesteryl esters and free fatty acids) were extracted from adipocyte cell pellets, using a method by Lowenstein et al. [18]. In summary, adipocytes were saponified in 200-proof ethanol and $30 \% \mathrm{KOH}(\mathrm{w} / \mathrm{v})$ in a $1: 1$ volume overnight on a $70{ }^{\circ} \mathrm{C}$ heating block. Samples were acidified with $\mathrm{HCl}$, fatty acids were extracted three times with petroleum ether, then air dried. Fatty acids were derivatized as methyl esters using $0.5 \mathrm{~N}$ methanolic $\mathrm{HCl}$, dried under a nitrogen stream, and subsequently reconstituted with hexane for gas chromatography/mass spectrometry (GC/MS) analysis.

\section{Gas chromatography/mass spectrometry}

GC/MS analysis was carried out using a HewlettPackard model 5973 selective mass detector connected to a model 6890 gas chromatograph. Fatty acids were analyzed as their methyl esters. Fatty acids were separated on the gas chromatograph with a Bpx70 column (30-m length, 250- $\mu \mathrm{m}$ diameter, $0.25-\mu \mathrm{m}$ film thickness) from SGE, Inc. (Austin, TX). The GC conditions were: helium flow rate, $1 \mathrm{ml} / \mathrm{min}$; initial oven temperature, $150{ }^{\circ} \mathrm{C}$; temperature was programmed to increase at $3{ }^{\circ} \mathrm{C} / \mathrm{min}$ to a final temperature of $221{ }^{\circ} \mathrm{C}$. The expected retention times under these conditions for palmitate, palmitoleate, stearate, oleate, and vaccenate (the major fatty acids of interest in this study) were as follows: $6.5,7.1,9.4$, 10.0, and $10.1 \mathrm{~min}$, respectively. Mass spectra of fatty acids were acquired using electron impact ionization and selective ion monitoring. Ion clusters monitored for isotopomer quantitation were mass-to-charge ratio $(\mathrm{m} / \mathrm{z}) 270$ for palmitate $(16: 0), \mathrm{m} / \mathrm{z} 268$ for palmitoleate $(16: 1), \mathrm{m} / \mathrm{z} 298$ for stearate (18:0), m/z 264 for oleate $(18: 1 \mathrm{n}-9)$ and vaccenate $(18: 1 \mathrm{n}-7)$. Distribution of the mass isotopomers was determined from the 
spectral data using a method previously described by Lee et al. [19] that corrects for the contribution of derivatizing agent and ${ }^{13} \mathrm{C}$ natural abundance to the mass isotopomer distribution of the compound of interest. Each compound of interest is composed of the sum of isotopomer peaks within a cluster. The resulting mass isotopomer distribution was expressed in molar fractions $(\mathrm{m} 0, \mathrm{~m} 1, \mathrm{~m} 2, \mathrm{~m} 3$, etc.) corresponding to the fraction of molecules that contain $0,1,2,3, \ldots{ }^{13} \mathrm{C}$ substitutions.

\section{Fatty acid profile}

The overall fatty acid profile was determined by calculation of the area under the respective gas chromatogram peaks for each main fatty acid of interest as a percent of total fatty acids. The percent of total for each fatty acid represents the total relative abundance (labeled plus unlabeled).

Fraction of new synthesis (FNS) over 24 hours, acetyl-CoA enrichment, and percent glucose contribution to de novo synthesis

The FNS represents the new fatty acids produced during the 24 hour experimental period, and is expressed as a percentage of total for each fatty acid. $\mathrm{M}+2$ and $\mathrm{M}+4$ isotopomers result from $\mathrm{U}^{13} \mathrm{C}$-glucose incorporation into palmitate in de novo lipogenesis, thereby allowing calculation of the precursor acetyl-CoA enrichment from the consecutive mass isotopomer ratio $\mathrm{m} 4 / \mathrm{m} 2$, and subsequent determination of the FNS. The FNS of stearate was determined from the $\mathrm{m} 6 / \mathrm{m} 4$ ratio to minimize potential chain elongation effects on the $\mathrm{m} 4 / \mathrm{m} 2$ ratio. Percent contribution to de novo lipogenesis (percent contribution to de novo synthesis of palmitate, the main product of de novo lipogenesis) from glucose was calculated based on the acetyl-CoA enrichment, and 50\% isotope enrichment of the medium glucose [20].

\section{Medium glucose determination}

Glucose concentration was determined on cell culture medium using the Glucose HK Assay Kit (Sigma) according the manufacturer's instructions, using spectrophotometry. Glucose content was calculated for the volume of medium used for each culture. Glucose consumption from the medium was then determined indirectly by subtracting the glucose content of the posttreatment medium from the glucose content of the original pretreatment medium.

\section{Tricarboxylic acid (TCA) cycle studies}

To determine the contribution of the TCA cycle to fatty acid synthesis, the relative contributions from the pyruvate carboxylase (PC) and pyruvate dehydrogenase (PDH) pathways were studied through determination of glutamate fragment enrichment [21]. Pyruvate carboxylase (PC) contributes toward production of gluconeogenic precursors through formation of oxaloacetate (OAA). PDH activity produces acetyl-CoA which can be used for fatty acid synthesis through formation of citrate. Downstream of citrate, glutamate equilibrates with $\alpha$-ketoglutarate [22], and reflects different fragment enrichment patterns based on contributions from the PC and PDH pathways. Glutamate was extracted from $1 \mathrm{ml}$ samples of medium by previously published methods using ion exchange columns [21,23]. Glutamate was derivatized as its n-trifluoroacetyl-n-butylester and analyzed by GC/MS using electron impact ionization. Two fragments were identified and the $\Sigma \mathrm{mn}$ enrichment (fraction of ${ }^{13} \mathrm{C}$ atoms/mole) was determined for each. The fragments were analyzed for the $\mathrm{m} 2$ isotopomer enrichment (m2): $\mathrm{m} / \mathrm{z}$ 152, representing the C2-C4 fragment (pyruvate carboxylase), and $\mathrm{m} / \mathrm{z} 198$, representing the $\mathrm{C} 2-\mathrm{C} 5$ fragment (pyruvate carboxylase + pyruvate dehydrogenase). The pyruvate carboxylase/pyruvate dehydrogenase $(\mathrm{PC} / \mathrm{PDH})$ ratio was determined by calculating (m2 of 152)/[(m2 of 198)-(m2 of 152)] to evaluate for differences in glucose metabolism via pyruvate entrance into the TCA cycle. When entry of glucose into the TCA cycle via $\mathrm{PC}$ and $\mathrm{PDH}$ are equal, the $\mathrm{PC} / \mathrm{PDH}$ ratio is approximately 1 . A decrease in the ratio suggests a relative increase in PDH activity, while an increase in the ratio suggests increased PC activity.

\section{Statistical analysis}

Statistical analysis was performed using SigmaStat software. Comparisons were made by ANOVA 1) within each group (Control or SGA) between the basal state and the treated states with increasing dose of PPAR $\gamma$ modulator, and also 2) between the two groups under the same treatment conditions. Fatty acid profile and stable isotope data were analyzed among treated and untreated Control and SGA groups by two-way ANOVA, followed by pair-wise multiple comparisons using Tukey's test. Parametric data are presented as mean \pm the standard error of the mean. Data that failed normality testing was analyzed by Kruskal-Wallis ANOVA on ranks, with pair-wise comparisons using Mann-Whitney testing. The non-parametric data (individual $\mathrm{PC}$ and PDH data) are therefore presented as the median with the $25-75 \%$ interquartile ranges. For comparison purposes, the PC/PDH ratio is also presented as the median with the $25-75 \%$ interquartile ranges.

\section{Results}

Preadipocyte proliferation and adipocyte oil red 0 staining

At both p1 and p21, SGA demonstrated increased preadipocyte proliferation and increased adipocyte lipid content as compared to Controls. With age, preadipocyte proliferation decreased while adipocyte lipid ORO 
staining increased in both Control and SGA adipose tissue (Figures 1, 2).

Adipogenic and lipogenic transcription factors at $\mathrm{p} 1$ At baseline, unstimulated SGA adipocytes demonstrated increased PPAR $\gamma$ protein expression, but comparable SREBP1 expression as compared to Controls (Figures 3A).

With rosiglitazone, Control and SGA adipocytes responded to low and high doses with marked increases in PPAR $\gamma$, and moderate, though significant, increases in SREBP1 (Figure 3A). The responses to both doses were similar in each group. Furthermore, SGA adipocytes maintained higher expression of PPAR $\gamma$, but similar SREBP1 expression as the Control adipocytes.

\section{Adipogenic and lipogenic transcription factors adipocyte phenotype at p21}

At baseline, SGA adipocytes continued to show upregulated PPAR $\gamma$, now accompanied by increased expression of SREBP1 (Figure 3B).

With rosiglitazone treatment, Control adipocytes responded to both (low and high) doses, demonstrating significant increases in PPARY and SREBP1 expression (Figure 3B). In contrast, SGA adipocytes responded at only the higher dose of rosiglitazone treatment with further upregulation of PPAR $\gamma$ and SREBP1 expression, thereby maintaining increased expression over Controls at only this dose.

After observing that p21 SGA adipocytes were responsive to only the higher dose of rosiglitazone, stable isotope studies were undertaken during high-dose rosiglitazone treatment.

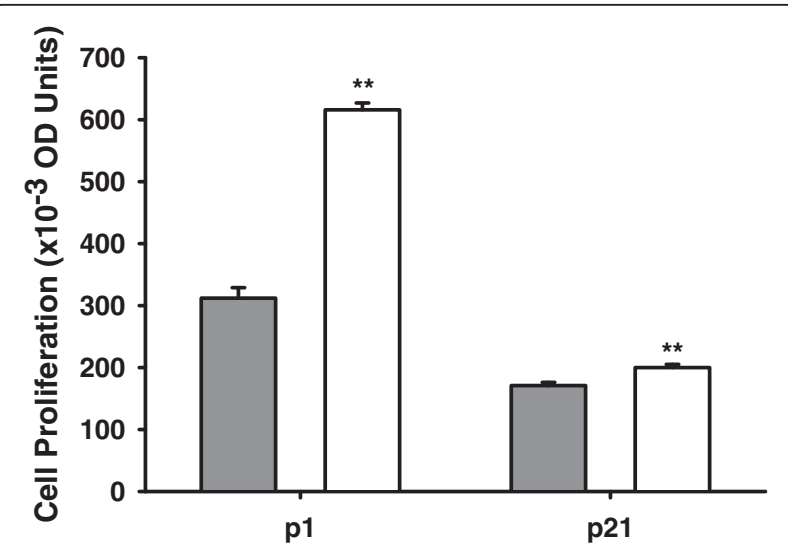

Figure 1 Cell proliferation in Control and SGA offspring preadipocytes. Gray bars represent Controls, and white bars represent SGA. Cell proliferation decreased with age in both groups. SGA demonstrated increased cell proliferation compared with Controls at both $p 1$ and $p 21,{ }^{* *} p<0.01$.

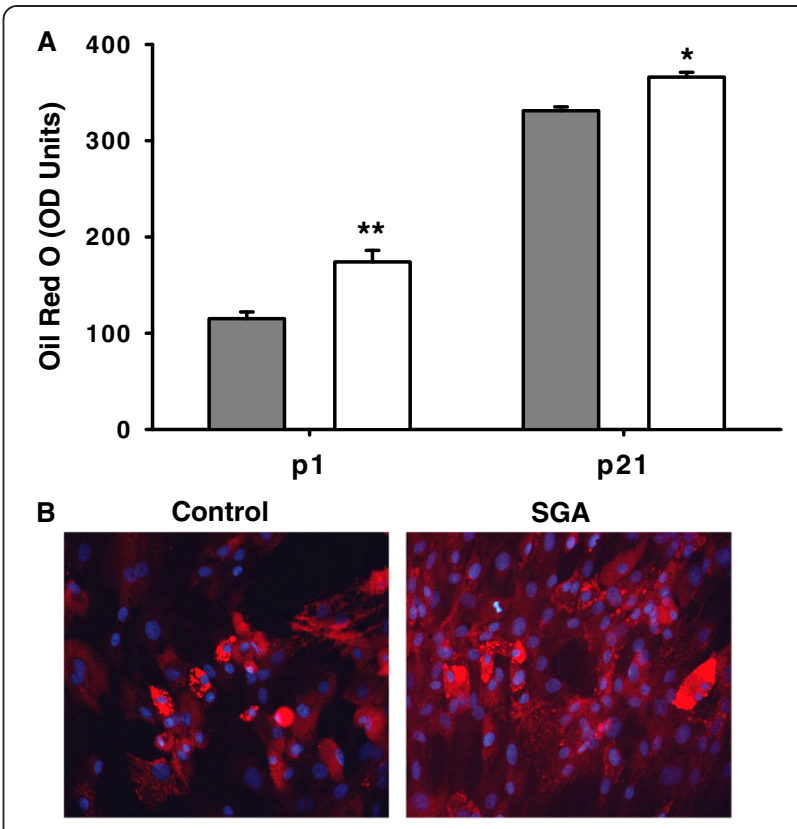

Figure 2 Oil Red O (ORO) staining in Control and SGA adipocytes. Gray bars represent Controls, and white bars represent SGA. (A) SGA adipocytes exhibit greater triglyceride accumulation than Controls at both p1 and p21. (B) SGA adipocytes at p21 demonstrate increased lipid accumulation at 40X magnification by ORO with DAPI staining. ${ }^{*} p<0.05,{ }^{* *} p<0.01$.

Fatty acid metabolism and response to $10 \mu \mathrm{M}$ rosiglitazone

Fatty acid profile

At baseline, SGA demonstrated a similar overall fatty acid profile as compared with Controls (Table 1). In response to rosiglitazone, Control, but not SGA adipocytes, increased the stearate percent of total, which includes preexisting and newly made stearate (Table 1).

\section{FNS over 24 hours}

At baseline, SGA had higher new synthesis (FNS) of palmitate, palmitoleate, stearate, and vaccenate over 24 hours (Figure 4).

In response to rosiglitazone (Figure 4), both Controls and SGA increased the FNS of palmitate and palmitoleate. Notably, Controls decreased the FNS stearate with rosiglitazone, while SGA did not respond. Overall, SGA maintained higher FNS rates of these three fatty acids (palmitate, palmitoleate, and stearate) than Controls. FASN expression, which was increased at baseline in SGA, also increased in response to rosiglitazone (Figure 4 inset).

\section{Glucose consumption studies}

At baseline, SGA adipocytes consumed more glucose over 24 hours (Table 2) than Controls. In both groups, glucose consumption was augmented by rosiglitazone 


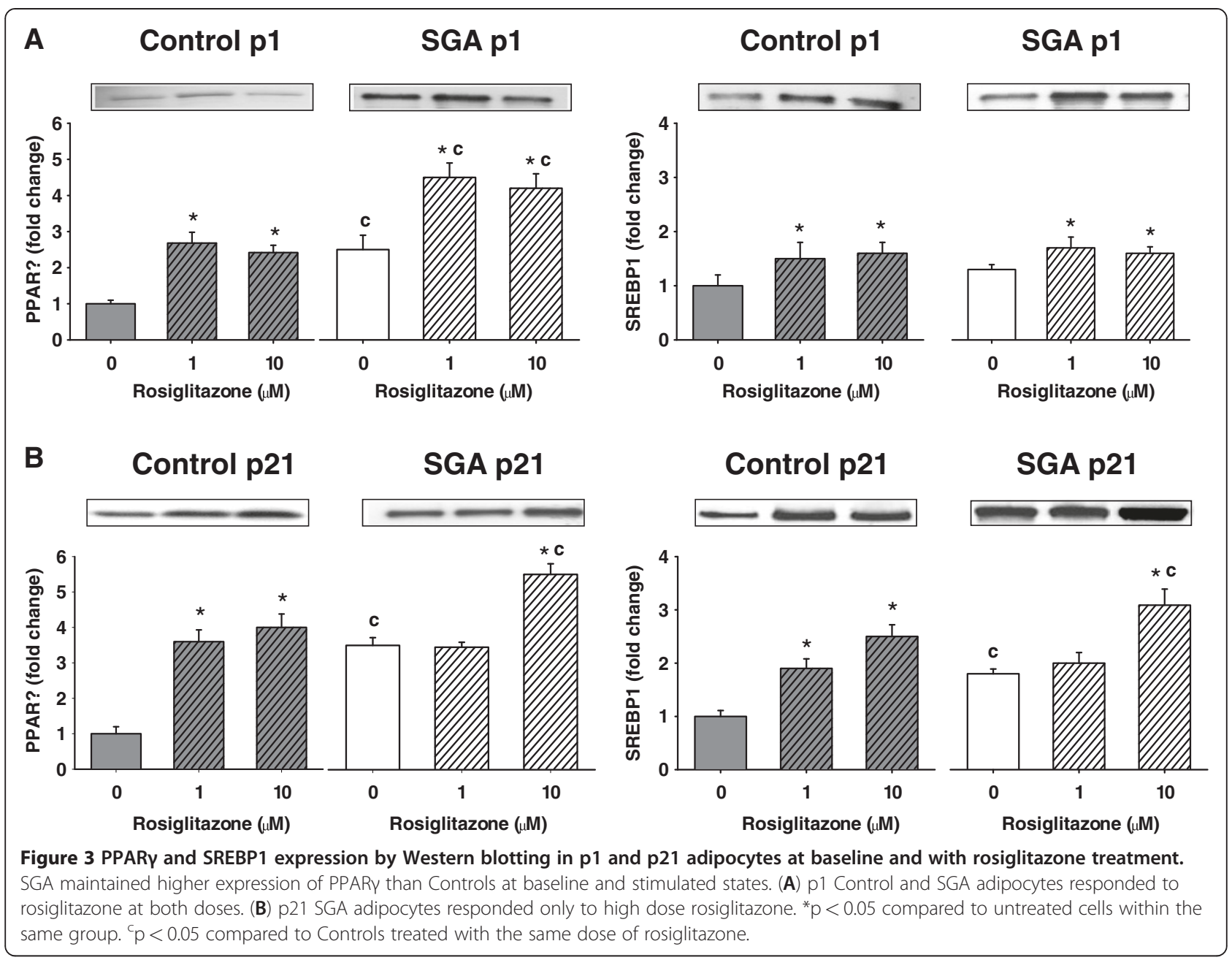

treatment (Table 2), but increased more in SGA than in Controls (+interaction).

\section{Acetyl-CoA enrichment}

At baseline, SGA demonstrated increased acetyl-CoA enrichment as well as increased percentage of $\mathrm{U}^{13} \mathrm{C}$ -

Table 1 Profile of major fatty acids, at baseline and with rosiglitazone

Fatty Acids Percent of Total (\%)

\begin{tabular}{|c|c|c|c|c|}
\hline & Control & & SGA & \\
\hline Fatty Acid & Baseline & Rosiglitazone & Baseline & Rosiglitazone \\
\hline Palmitate 16:0 & $36.4 \pm 1.5$ & $37.9 \pm 1.1$ & $37.5 \pm 0.7$ & $37.7 \pm 2.4$ \\
\hline Palmitoleate $16: 1 n-7$ & $7 \quad 3.4 \pm 0.1$ & $3.09 \pm 0.04$ & $3.4 \pm 0.1$ & $3.3 \pm 0.3$ \\
\hline Stearate 18:0 & $22.6 \pm 0.9$ & $29.1 \pm 0.9^{*}$ & $20.8 \pm 0.6$ & $22.4 \pm 1.4^{c}$ \\
\hline Oleate $18: 1 n-9$ & $19.7 \pm 0.7$ & $17.4 \pm 0.4$ & $20.0 \pm 0.3$ & $20.1 \pm 1.2$ \\
\hline Vaccenate $18: 1 n-7$ & $12.0 \pm 0.5$ & $10.4 \pm 0.2$ & $13.2 \pm 0.3$ & $12.1 \pm 0.9$ \\
\hline
\end{tabular}

glucose contribution to palmitate de novo synthesis (Figure 5). With rosiglitazone treatment, these measures were enhanced in both groups, with SGA maintaining higher levels than Controls. Greater increases were observed in SGA (+interaction) than in Controls.

\section{TCA cycle activities}

To elucidate potential TCA cycle contributions to the increased acetyl-CoA enrichment, glutamate enrichment was studied to determine the relative pyruvate carboxylase and pyruvate dehydrogenase activities. SGA demonstrated increased entry of $\mathrm{U}^{13} \mathrm{C}$-glucose into the TCA cycle in SGA based on the higher $\Sigma \mathrm{mn}$ enrichment (fraction of ${ }^{13} \mathrm{C}$ atoms/mole) in both the glutamate $\mathrm{C} 2-4$ and C2-5 fragments (Table 3A). SGA demonstrated a lower $\mathrm{PC} / \mathrm{PDH}$ ratio as compared with Controls (Table $3 \mathrm{~B}$ ), suggesting higher $\mathrm{PDH}$ activity relative to $\mathrm{PC}$ activity, possibly leading to increased acetyl-CoA production for lipogenesis.

In response to rosiglitazone, both groups increased enrichment $(\mathrm{Smn})$ in the $\mathrm{C} 2-4$ and $\mathrm{C} 2-5$ glutamate fragments 


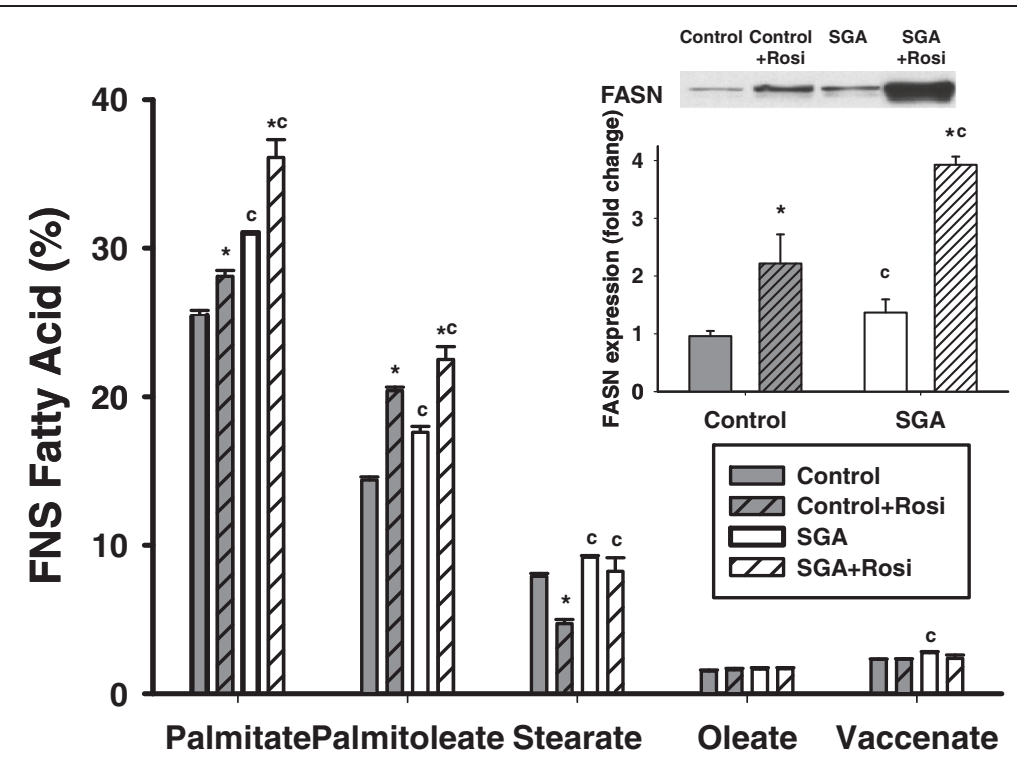

Figure 4 Fraction of new synthesis (FNS) of fatty acids and FASN expression in Controls and SGA at baseline and with $10 \mu \mathrm{M}$ rosiglitazone treatment. The data is presented as the mean percentage or mean fold-change in expression \pm the standard error of the mean. SGA demonstrated increased FNS palmitate and palmitoleate at baseline and with rosiglitazone when compared to Controls, with a similar pattern in FASN protein expression (inset). ${ }^{*} p<0.05$ compared to untreated state within the same group, ${ }^{c} p<0.05$ compared to Controls receiving the same treatment.

(Table 3A). Controls exhibited a decrease in the PC/PDH ratio while SGA increased it to a level similar to Controls (Table 3B), with a significant interaction between SGA and rosiglitazone. The increase of the $\mathrm{PC} / \mathrm{PDH}$ ratio suggested that SGA shifted its glucose entry to be more balanced between $\mathrm{PC}$ and $\mathrm{PDH}$, but this change could not be attributed to significant changes in either the $\mathrm{PC}$ or $\mathrm{PDH}$ enrichment beyond the changes expected based on the $\Sigma \mathrm{mn}$.

\section{BADGE treatment}

At p1, both Control and SGA adipocytes showed moderate decreases in PPAR $\gamma$ and SREBP1 expression at low dose BADGE, then further decreases with high dose BADGE. As before, SGA adipocytes continued to exhibit higher expression of PPAR (Figure 6A).

At p21, Control and SGA adipocytes differed in their response to BADGE treatment. While Control adipocytes responded with downregulation of PPAR $\gamma$ and SREBP1, SGA adipocytes were completely unresponsive

Table 2 Glucose consumption from cell culture medium in $\mathbf{2 4}$ hours at baseline or with rosiglitazone

\begin{tabular}{lll}
\hline & $\begin{array}{l}\text { Glucose Consumption } \\
(\mathbf{m g} / \mathbf{m L} \text { of culture medium) }\end{array}$ \\
\hline & Control & SGA \\
\hline Baseline & $0.99 \pm 0.02$ & $1.23 \pm 0.01^{c}$ \\
\hline Rosiglitazone Treatment & $1.14 \pm 0.01^{*}$ & $1.45 \pm 0.01^{* \mathrm{ci}}$ \\
\hline${ }^{\mathrm{c}} \mathrm{p}<0.05$ compared with Control receiving the same treatment. \\
* $\mathrm{p}<0.05$ compared to the untreated state within the same group. $^{\mathrm{i}}$ interaction with rosiglitazone.
\end{tabular}

(Figure 6B). Due to the lack of response, the fatty acid metabolic studies were not repeated with BADGE.

\section{Discussion}

This study demonstrates that SGA adipocytes are intrinsically programmed by maternal malnutrition as evident in the principal findings of: (1) enhanced adipogenesis through upregulated PPAR $\gamma$ expression, and (2) enhanced lipogenesis through upregulated SREBP1 and FASN expression, with increased glucose-mediated fatty acid synthesis. Importantly, SGA adipocytes are overall responsive to PPAR $\gamma$ activator-ligand, but are selectively responsive at p21 to the higher dose of PPAR $\gamma$ activator-ligand, with pathway-specific effects on fatty acid synthesis. In contrast, SGA adipocytes at p21 are unresponsive to PPAR $\gamma$ inhibitor-ligand. Together, these results indicate that the SGA adipocyte phenotype is programmed in utero, as demonstrated by enhanced preadipocyte proliferation and lipid accumulation at $\mathrm{p} 1$. Due to resistance to treatment at p21, therapeutic strategies to prevent the obesogenic adipocyte phenotype should be considered during the nursing period prior to onset of the resistance.

Consistent with previous studies [24,25], preadipocyte proliferation declined and adipocyte fat accumulation amplified with age in both the Control and SGA offspring. Notably, despite SGA growth-restriction at birth, the SGA adipose tissue exhibited increased preadipocyte proliferation with enhanced fat accumulation, consistent with an obese phenotype. The upregulated transcription factors (PPAR $\gamma$, SREBP1) together with increased glucose- 


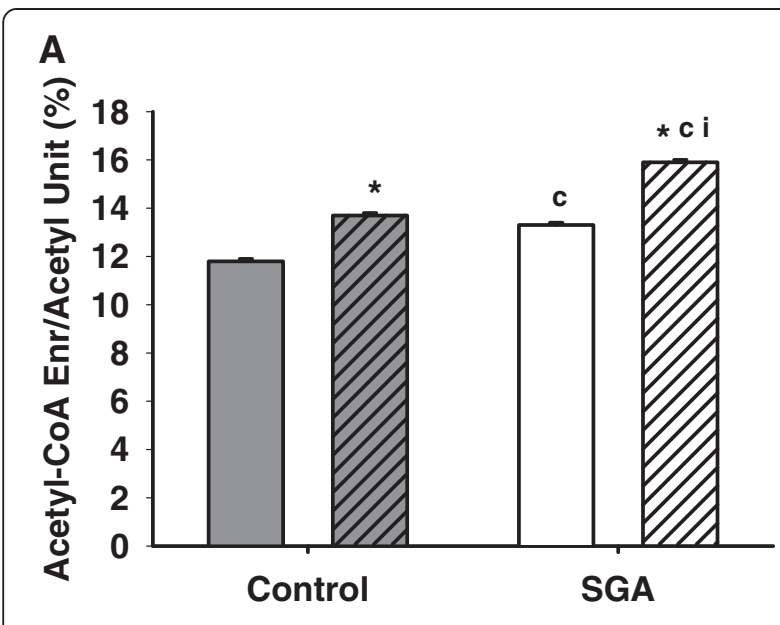

B

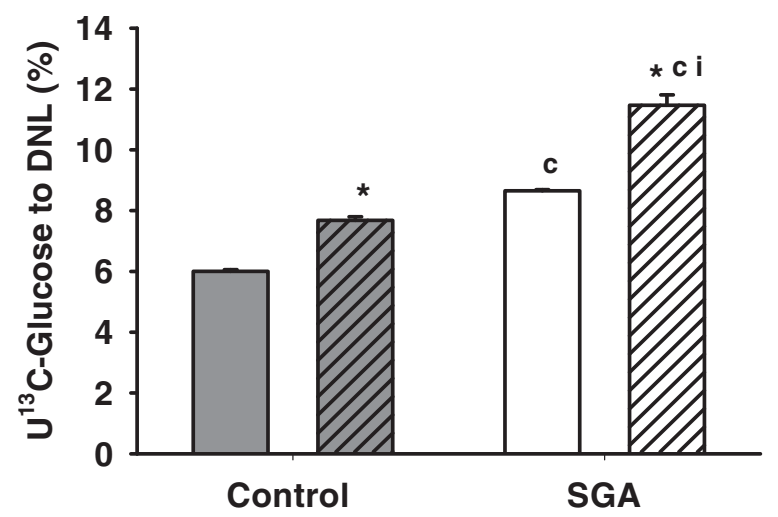

Figure 5 Acetyl-CoA enrichment and $U^{13} \mathrm{C}$-glucose contribution to de novo lipogenesis (DNL). Gray bars represent Controls, and white bars represent SGA. Solid bars represent adipocytes untreated at baseline, and hatched bars represent adipocytes treated with $10 \mu \mathrm{M}$ rosiglitazone. (A) SGA adipocytes exhibited increased acetyl$\mathrm{CoA}$ enrichment, and therefore, (B) increased $\mathrm{U}^{13} \mathrm{C}$-glucose contribution to de novo synthesis of palmitate. There was a significant interaction between SGA and rosiglitazone for each of these measures. ${ }^{*} p<0.05$ compared to untreated state within the same group, ${ }^{C} p<0.05$ compared to Controls receiving the same treatment, ${ }^{i}$ interaction with rosiglitazone.

mediated fatty acid synthesis likely facilitate enhanced adipogenesis and lipogenesis in SGA offspring. Moreover, these changes are evident and persistent at postnatal age p21 in SGA offspring. Increased risk for obesity in SGA has been well-described, with SGA humans exhibiting increased visceral adiposity [26] or increased clinical features of abdominal adiposity such as waist circumference [1]. Although obesity may not manifest until adulthood, our data in nursing SGA pups demonstrating increased hyperplasia and hypertrophy along with enhanced fatty acid de novo synthesis indicates the early presence of abnormalities that may contribute toward the propensity for future fat accumulation.

The differential response to rosiglitazone revealed pathway-specific and age-specific changes in lipogenesis between Controls and SGA. Expression of adipogenic and lipogenic factors was stimulated in both groups at p1. At p21, however, Controls demonstrated increased responsiveness with dose increase, while SGA responded from its basally increased expression to only high-dose rosiglitazone. The selective response of SGA to the highdose rosiglitazone indicates a decline in responsiveness by the end of the nursing period. Nonetheless, the decreased responsiveness of SGA at p21 indicated changes leading toward an obese phenotype as evident by the fatty acid synthesis data. Further studies are needed in offspring from $\mathrm{p} 0$ to $\mathrm{p} 21$ to determine the factors in the metabolic milieu that may ultimately lead to p21 rosiglitazone resistance.

In Controls and SGA, rosiglitazone treatment led to increased FNS of palmitate and palmitoleate, with a similar pattern of increase in FASN expression. However, the two groups demonstrated a differential response in 18-carbon stearate synthesis. The Control group increased its percent of total of stearate in response to rosiglitazone, despite decreasing the FNS stearate, suggesting decreased stearate turnover as a normal response to rosiglitazone. In contrast, SGA adipocytes did not decrease the FNS stearate in response to rosiglitazone, and maintained the new synthesis rate, suggesting abnormal resistance to modulation in the 18-carbon fatty acid synthesis pathway. The difference in FNS stearate versus palmitate response to rosiglitazone supports possibilities of differential regulation and/or compartmentalization of the 16-carbon versus 18-carbon fatty acid synthesis pathways [20]. Since the FASN expression reflected the FNS palmitate response to rosiglitazone in both groups, the stearate responses to rosiglitazone are likely mediated by a posttranslational mechanism. Whether these differences in fatty acid metabolism after rosiglitazone treatment impact adipocyte function in SGA is not known from our study, but would be of interest in the setting of longterm treatment.

Rosiglitazone improves blood glucose control in humans with diabetes through increased glucose uptake by muscle and adipose tissue [27]. Therefore, we used the stable isotope $\mathrm{U}^{13} \mathrm{C}$-glucose to determine the relationship between the fatty acid synthesis pathways and glucose metabolism in the adipocytes. In fact, our data demonstrate that SGA adipocytes use more glucose toward fatty acid synthesis (increased acetyl-CoA enrichment). Moreover, SGA demonstrated an augmentation of response to rosiglitazone (+interaction) possibly through a programmed increase in sensitivity to glucose abundance. Correspondingly, SGA demonstrated an increased percent contribution of $\mathrm{U}^{13} \mathrm{C}$-glucose to de novo synthesis with rosiglitazone treatment. These findings led to investigations into the PC and PDH-mediated glucose entry pathways into the TCA cycle. 
Table 3 Relative PC and PDH activities determined from adipocyte exposure to $U^{13}$-glucose

A.

\begin{tabular}{lllll}
\hline & \multicolumn{2}{l}{ Imn C2-4 fragment (mean \pm SEM) } & \multicolumn{2}{l}{ Imn C2-5 fragment (mean \pm SEM) } \\
\hline Baseline & Control & SGA & Control & SGA \\
\hline Rosi Treatment & $0.056 \pm 0.003$ & $0.073 \pm 0.001^{C}$ & $0.060 \pm 0.003$ & $0.084 \pm 0.001^{C}$ \\
\hline
\end{tabular}

B.

\begin{tabular}{lllllll}
\hline & \multicolumn{2}{l}{$\begin{array}{l}\text { PC (m2 152) } \\
\text { (median with 25-75\%) }\end{array}$} & \multicolumn{2}{l}{$\begin{array}{l}\text { PDH (m2 198 - m2 152) } \\
\text { (median with 25-75\%) }\end{array}$} & \multicolumn{2}{l}{$\begin{array}{l}\text { PC/PDH } \\
\text { (median with 25-75\%) }\end{array}$} \\
\hline Control & SGA & Control & SGA & Control & SGA & Control \\
\hline Baseline & $0.015(0.015-0.017)$ & $0.018(0.017-0.018)$ & $0.009(0.009-0.010)$ & $0.017(0.016-0.018)$ & $1.66(1.64-1.75)$ & $0.92(0.90-1.04)^{C}$ \\
\hline Rosi Treatment & $0.019(0.018-0.028)$ & $0.025(0.025-0.026)$ & $0.021(0.021-0.022$ & $0.021(0.021-0.022)$ & $1.10(1.10-1.19)^{*}$ & $1.17(1.14-1.18)^{* i}$ \\
\hline
\end{tabular}

$\mathrm{C}_{\mathrm{p}}<0.05$ compared with Control receiving the same treatment.

${ }^{*} p<0.05$ compared to the untreated state within the same group.

' interaction between SGA and rosiglitazone.

A) The $\Sigma m n$ representing the average fraction of ${ }^{13} \mathrm{C}$ atoms/molecule for the $\mathrm{C} 2-4$ fragment and C2-5 fragments are shown for cultures with and without rosiglitazone treatment. The data are presented as fractions of enrichment \pm the SEM. Enrichment increased with rosiglitazone treatment in both Controls and SGA, while SGA maintained higher levels than Controls regardless of treatment. B) The PC and PDH pathway contributions are represented based on glutamate fragment enrichments. These data are presented as the median with the $25-75 \%$ interquartile ranges. At baseline, SGA demonstrated a lower PC/PDH ratio than Controls. With rosiglitazone, Controls decreased the PC/PDH ratio, while SGA increased it.

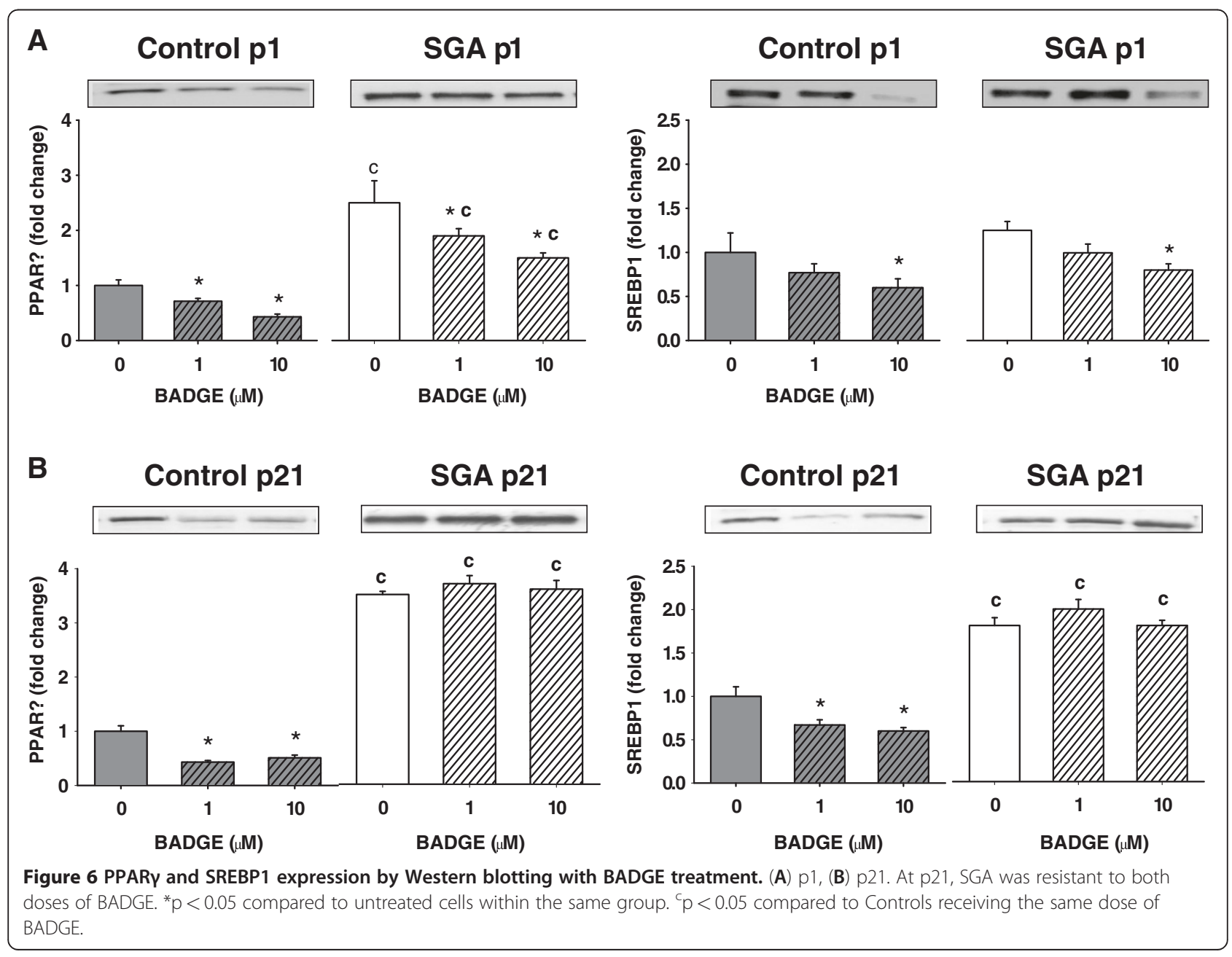


Analysis of the PC/PDH ratio was performed to determine how entry of glucose into the TCA cycle contributed to the increased acetyl-CoA enrichment in SGA. SGA adipocytes at baseline exhibited a lower PC/PDH ratio than Controls, suggesting that SGA adipocytes demonstrate enhanced glucose entry into the TCA cycle to produce acetyl-CoA for fatty acid synthesis via $\mathrm{PDH}$. Rosiglitazone treatment led to a decrease in the $\mathrm{PC} / \mathrm{PDH}$ ratio in Controls, supporting enhanced lipogenesis, while SGA adipocytes unexpectedly demonstrated an increased ratio. Changes in the separate PC and PDH enrichments were therefore examined relatively to changes in the $\Sigma \mathrm{mn}$. The $\Sigma \mathrm{mn}$ was increased in SGA at baseline and further increased with rosiglitazone in both glutamate fragments, with corresponding trends in PC and PDH $\mathrm{m} 2$ enrichment. However, the SGA PC enrichment increased by $39 \%$ with rosiglitazone treatment, while the PDH enrichment increased by only $29 \%$, resulting in a net increase in the $\mathrm{PC} / \mathrm{PDH}$ ratio. An alternative explanation for the increased acetyl-CoA enrichment in our study could be increased recycling of labeled palmitate, through oxidation and reassembly of labeled acetyl units in de novo synthesis. Additional factors may also be contributing to our findings, since the turnover of glutamate is low, and the percentage of glucose contribution to palmitate comprised only up to $6-12 \%$. The pathway of glucose utilization to produce palmitate is likely not direct [28], involving other pathways before these carbons are incorporated into fatty acids. However, this data illustrates the complex relationship that exists between fatty acid synthesis and glucose metabolism, which involve multiple metabolic pathways.

Control adipocytes responded to BADGE at both p1 and $\mathrm{p} 21$, with further decrease in expression of both PPARY and SREBP1 at the high dose. In contrast, SGA adipocytes demonstrated BADGE resistance at both doses of rosiglitazone, suggesting that stimulatory factors that are present cannot be overcome by BADGE at the experimental doses. It is unknown whether even a higher dose may have been effective.

Despite the current obesity epidemic, effective strategies for the prevention or treatment of obesity and related disorders are very limited. SGA adipocytes, previously adapted to the in utero environment of low energy resources, suddenly experience a relative nutrient excess with normal postnatal nutrition [29]. As a result, adipocytes of SGA offspring demonstrate enhanced cell proliferation, and lipogenesis, which may allow SGA offspring to catch-up in growth. The increased adipogenesis however, leads to adult obesity and insulin resistance. TZDs are used to improve insulin sensitivity, but they have also been studied for their potential to prevent insulin resistance [30,31]. Adipose tissue is the major site of action of TZDs. Further studies are needed to define the relationship between glucose utilization for fatty acid synthesis and insulin sensitivity, in order to determine if TZDs may be efficacious in preventing insulin resistance in SGA. Studies are also needed to determine how the 16carbon versus 18-carbon fatty acid synthesis pathways contribute to adipocyte phenotype in SGA, and whether differences in response of these pathways to rosiglitazone may interfere with its potential to prevent insulin resistance. If glucose-mediated lipogenesis contributes to insulin sensitivity in TZD treatment, then risks of further adipogenesis during TZD therapy must be weighed against potential benefits of glucose control.

\section{Conclusions}

SGA adipocytes at p1 and p21 exhibit enhanced adipogenesis and lipogenesis. p1 adipocytes respond to both activator-ligand and repressor-ligand treatment. However, p21 SGA adipocytes demonstrate pathway-specific responses to PPAR $\gamma$ activator-ligand treatment in fatty acid synthesis. p21 SGA adipocytes are also resistant to PPARY repressor-ligand treatment. PPAR $\gamma$ activatorligand treatment enhances SGA glucose-mediated lipogenesis. The complex relationship between fatty acid synthesis and glucose metabolic pathways should be further investigated in SGA adipocytes.

\section{Abbreviations}

SGA: Small-for-gestational age; PPARY: Peroxisome proliferator-activated receptor gamma; BADGE: Bisphenol-A diglycidyl ether;

TZD: Thiazolidinedione; ORO: Oil Red O; GC/MS: Gas chromatography/mass spectrometry; FNS: Fractional synthesis (or fraction of new synthesis); TCA: Tricarboxylic acid; PC: Pyruvate carboxylase; PDH: Pyruvate dehydrogenase; SREBP1: Sterol regulatory element binding protein 1; FASN: Fatty acid synthase; DMEM: Dulbecco's modified Eagle's medium; FBS: Fetal bovine serum; MTT: 3-(4,5-Dimethylthiazol-2-yl)-2,5diphenyltetrazolium bromide; Rosi: Rosiglitazone.

\section{Competing interests}

The authors have no competing interests to declare.

\section{Authors' contributions}

JKY participated in the study design, execution, analysis and interpretation of the fatty acid/stable isotope studies, and also drafted the manuscript. WPL participated in the fatty acid/stable isotope data analysis and interpretation, and contributed to manuscript preparation. MGR participated in manuscript preparation. $\mathrm{RHL}$ participated in manuscript preparation. GH established the adipocyte cultures, performed the MTT assay, ORO staining, and Western blotting, and contributed to manuscript preparation. JV performed Western blotting and contributed to manuscript preparation. MD participated in study design, data analysis and interpretation, and drafting of the manuscript. All authors read and approved the final manuscript.

\section{Acknowledgements}

We appreciate Linda Day, Stacy Behare, and Catherine S. Mao for their assistance in animal care. We thank Shu Lim and Paulin Wahjudi for technical assistance in glutamate analysis. J.K.Y. is supported by grant K23 DK08324 from the NIDDK and the Clinical Scholar Award from the Pediatric Endocrine Society. M.D. is supported by grant R01DK081756 from the NIDDK. The GC/ MS analyses were performed at the LABioMed at Harbor-UCLA Biomedical Mass Spectrometry Facility, which is supported by the UCLA CTSI through grant 1UL1-RR033176.

\section{Author details}

${ }^{1}$ Department of Pediatrics, Division of Endocrinology, Los Angeles Biomedical Research Institute at Harbor-UCLA Medical Center, David Geffen School of 
Medicine at UCLA, 1000 West Carson Street, Harbor Box 446, Torrance, CA 90509, USA. ²Department of Obstetrics and Gynecology, Los Angeles Biomedical Research Institute at Harbor-UCLA Medical Center, David Geffen School of Medicine at UCLA, 1000 West Carson Street, Harbor Box 446, Torrance, CA 90509, USA. ${ }^{3}$ Division of Neonatology, University of Utah School of Medicine, Williams Building, P.O. Box 581289, Salt Lake City, UT 84158, USA

Received: 12 April 2012 Accepted: 14 June 2012

Published: 22 June 2012

\section{References}

1. Ravelli AC, van Der Meulen JH, Osmond C, Barker DJ, Bleker OP: Obesity at the age of $50 \mathrm{y}$ in men and women exposed to famine prenatally. Am $J$ Clin Nutr 1999, 70:811-816.

2. Ravelli GP, Stein ZA, Susser MW: Obesity in young men after famine exposure in utero and early infancy. N Engl J Med 1976, 295:349-353.

3. Yee JK, Lee WN, Han G, Ross MG, Desai M: Organ-specific alterations in fatty acid de novo synthesis and desaturation in a rat model of programmed obesity. Lipids Health Dis 2011, 10:72.

4. Desai M, Gayle D, Babu J, Ross MG: Programmed obesity in intrauterine growth-restricted newborns: modulation by newborn nutrition. Am J Physiol Regul Integr Comp Physiol 2005, 288:R91-R96.

5. Desai M, Gayle D, Babu J, Ross MG: The timing of nutrient restriction during rat pregnancy/lactation alters metabolic syndrome phenotype. Am J Obstet Gynecol 2007, 196:e551-e557.

6. Desai M, Gayle D, Han G, Ross MG: Programmed hyperphagia due to reduced anorexigenic mechanisms in intrauterine growth-restricted offspring. Reprod Sci 2007, 14:329-337.

7. Desai M, Guang H, Ferelli M, Kallichanda N, Lane RH: Programmed upregulation of adipogenic transcription factors in intrauterine growthrestricted offspring. Reprod Sci 2008, 15:785-796.

8. Kim JB, Spiegelman BM: ADD1/SREBP1 promotes adipocyte differentiation and gene expression linked to fatty acid metabolism. Genes Dev 1996, 10:1096-1107.

9. Kahn SE, Haffner SM, Heise MA, Herman WH, Holman RR, Jones NP, Kravitz BG, Lachin JM, O'Neill MC, Zinman B, Viberti G: Glycemic durability of rosiglitazone, metformin, or glyburide monotherapy. N Engl J Med 2006, 355:2427-2443.

10. Albrektsen T, Frederiksen KS, Holmes WE, Boel E, Taylor K, Fleckner J: Novel genes regulated by the insulin sensitizer rosiglitazone during adipocyte differentiation. Diabetes 2002, 51:1042-1051.

11. Muhlhausler BS, Morrison $J \mathrm{~L}$, McMillen IC: Rosiglitazone increases the expression of peroxisome proliferator-activated receptor-gamma target genes in adipose tissue, liver, and skeletal muscle in the sheep fetus in late gestation. Endocrinology 2009, 150:4287-4294.

12. Kim SK, Park CK, Lee SY, Song JS, Park SH, Kim YK: Effects of Rosiglitazone on the Expression of PPAR-gamma and the Production of IL- 6 and IL-8 in Acute Lung Injury Model Using Human Pulmonary Epithelial Cells. Trop J Pharm Res 2011, 10:559-566.

13. Johnson JA, Trasino SE, Ferrante AW Jr, Vasselli JR: Prolonged decrease of adipocyte size after rosiglitazone treatment in high- and low-fat-fed rats. Obesity (Silver Spring) 2007, 15:2653-2663.

14. Ye JM, Dzamko N, Cleasby ME, Hegarty BD, Furler SM, Cooney GJ, Kraegen EW: Direct demonstration of lipid sequestration as a mechanism by which rosiglitazone prevents fatty-acid-induced insulin resistance in the rat: comparison with metformin. Diabetologia 2004, 47:1306-1313.

15. Oakes ND, Thalen PG, Jacinto SM, Ljung B: Thiazolidinediones increase plasma-adipose tissue FFA exchange capacity and enhance insulin-mediated control of systemic FFA availability. Diabetes 2001 50:1158-1165

16. Wright HM, Clish CB, Mikami T, Hauser S, Yanagi K, Hiramatsu R, Serhan CN, Spiegelman BM: A synthetic antagonist for the peroxisome proliferatoractivated receptor gamma inhibits adipocyte differentiation. J Biol Chem 2000, 275:1873-1877.

17. Kota BP, Huang TH, Roufogalis BD: An overview on biological mechanisms of PPARs. Pharmacol Res 2005, 51:85-94.

18. Lowenstein JM, Brunengraber $H$, Wadke M: Measurement of rates of lipogenesis with deuterated and tritiated water. Methods Enzymol 1975, 35:279-287
19. Lee WN, Byerley LO, Bergner EA, Edmond J: Mass isotopomer analysis: theoretical and practical considerations. Biol Mass Spectrom 1991 20:451-458.

20. Yee JK, Mao CS, Hummel HS, Lim S, Sugano S, Rehan VK, Xiao G, Lee WN: Compartmentalization of stearoyl-coenzyme A desaturase 1 activity in HepG2 cells. J Lipid Res 2008, 49:2124-2134.

21. Lee WN, Edmond J, Bassilian S, Morrow JW: Mass isotopomer study of glutamine oxidation and synthesis in primary culture of astrocytes. Dev Neurosci 1996, 18:469-477.

22. Des Rosiers C, Lloyd S, Comte B, Chatham JC: A critical perspective of the use of (13)C-isotopomer analysis by GCMS and NMR as applied to cardiac metabolism. Metab Eng 2004, 6:44-58.

23. Bartnik BL, Hovda DA, Lee PW: Glucose metabolism after traumatic brain injury: estimation of pyruvate carboxylase and pyruvate dehydrogenase flux by mass isotopomer analysis. J Neurotrauma 2007, 24:181-194.

24. Kirkland JL, Hollenberg CH, Gillon WS: Age, anatomic site, and the replication and differentiation of adipocyte precursors. Am J Physiol 1990, 258:C206-C210.

25. Honma T, Yanaka M, Tsuduki T, Ikeda I: Increased lipid accumulation in liver and white adipose tissue in aging in the SAMP10 mouse. J Nutr SCi Vitaminol (Tokyo) 2011, 57:123-129.

26. Ibanez L, Lopez-Bermejo A, Suarez L, Marcos MV, Diaz M, de Zegher F: Visceral adiposity without overweight in children born small for gestational age. J Clin Endocrinol Metab 2008, 93:2079-2083.

27. Yki-Jarvinen H: Thiazolidinediones. N Engl J Med 2004, 351:1106-1118.

28. Collins JM, Neville MJ, Pinnick KE, Hodson L, Ruyter B, van Dijk TH, Reijngoud DJ, Fielding MD, Frayn KN: De novo lipogenesis in the differentiating human adipocyte can provide all fatty acids necessary for maturation. J Lipid Res 2011, 52:1683-1692.

29. Gluckman PD, Hanson MA: Living with the past: evolution, development and patterns of disease. Science 2004, 305:1733-1736.

30. Hevener AL, Reichart D, Janez A, Olefsky J: Thiazolidinedione treatment prevents free fatty acid-induced insulin resistance in male wistar rats. Diabetes 2001, 50:2316-2322.

31. Chung SS, Kim M, Youn BS, Lee NS, Park JW, Lee IK, Lee YS, Kim JB, Cho YM, Lee HK, Park KS: Glutathione peroxidase 3 mediates the antioxidant effect of peroxisome proliferator-activated receptor gamma in human skeletal muscle cells. Mol Cell Biol 2009, 29:20-30.

doi:10.1186/1743-7075-9-62

Cite this article as: Yee et al:: Peroxisome proliferator-activated receptor gamma modulation and lipogenic response in adipocytes of small-forgestational age offspring. Nutrition \& Metabolism 2012 9:62.

\section{Submit your next manuscript to BioMed Central and take full advantage of:}

- Convenient online submission

- Thorough peer review

- No space constraints or color figure charges

- Immediate publication on acceptance

- Inclusion in PubMed, CAS, Scopus and Google Scholar

- Research which is freely available for redistribution 